[0212-7199 (2004) 21: 11; pp 554-556] Copyright (C) 2004 ARAN EDICIONES, S.L.

AN. MED. INTERna (Madrid) Vol. 21, N. ${ }^{\circ} 11$, pp. 554-556, 2004

\title{
Neumonía por Nocardia farcinica en paciente con EPOC
}

\author{
F. DEL CAMPO MATÍAS, J. L. PÉREZ CASTRILLÓN', J. L. GONZÁLEZ GARCÍA², \\ P. PÉREZ PASCUAL ${ }^{3}$
}

Servicios de Neumología, ${ }^{1}$ Medicina Interna, ${ }^{2}$ Radiodiagnóstico y de ${ }^{3}$ Microbiología. Hospital Universitario Río Hortega. Valladolid

\section{RESUMEN}

La Nocardia farcinica es una infección infrecuente, que tiene tendencia a aparecer en pacientes con factores de riesgo predisponentes, fundamentalmente inmunodeprimidos, aunque también ha sido descrita en pacientes inmunocompetentes. Su importancia como patógeno emergente ha sido reconocida tan sólo en los últimos años. Afecta predominantemente al pulmón, siendo indistinguible en cuanto a su presentación clínica y radiológica del resto de las neumonías. El fracaso terapéutico es la razón principal de su sospecha.

PALABRAS CLAVE: Neumonía por Nocardia farcinica.
NOCARDIA FARCINICA PNEUMONIAE IN CHRONIC OBSTRUCTIVE PULMONARY DISEASE PATIENT

\section{ABSTRACT}

Nocardia farcinica is an infrequent infection that usually appears in patients with predisposing conditions, especially in immunosuppressed patients, although it has also been found in healthy individuals. It's importance as a new pathogen has been recognized only in recent years. Mainly, it affects the lung, indistinguishable from other types of pneumonia in the clinical and radiological characteristics. The main reason for detection is therapeutic failure.

KEY WORDS: Nocardia farcinica pneumoniae.

Del Campo Matías F, Pérez Castrillón JL, González García JI, Pérez Pascual P. Neumonía por Nocardia farcinica en paciente con EPOC. An Med Interna (Madrid) 2004; 21: 554-556.

\section{INTRODUCCIÓN}

La nocardiosis pulmonar es una infección respiratoria, descrita habitualmente en pacientes inmunodeprimidos, sin embargo puede aparecer en pacientes inmunocompetentes o que presentan patología pulmonar subyacente, no siendo excepcional la infección pulmonar por nocardia en pacientes con EPOC. El diagnóstico requiere una alta sospecha clínica, dado que sus características clínicas, radiológicas y analíticas son indistinguibles de cualquier proceso neumónico, siendo el fracaso terapéutico la principal razón de su consideración. El diagnostico puede ser fácilmente establecido a través del cultivo de esputo. La nocardia asteroides es el tipo de nocardia más frecuentemente implicado. Sin embargo, la mejora de las técnicas diagnósticas ha permitido un mayor número de aislamientos de nocardia farcínica, hecho importante dado su diferente comportamiento clínico y respuesta al tratamiento antibiótico que presenta en relación con el resto de las nocardias.

\section{CASO APORTADO}

Paciente de 77 años de edad, que es ingresado como consecuencia de presentar un cuadro de tres días de evolución caracterizado por dolor torácico, de aparición brusca y características pleuríticas, junto con incremento de su tos habitual, expectoración purulenta y fiebre de $38^{\circ} \mathrm{C}$. A la exploración física destacaba una ligera taquipnea y a la auscultación pulmonar crepitantes en campo medio e inferior de hemitórax derecho.

Entre sus antecedentes personales destacaba estar diagnosticado de EPOC severo desde hacía quince años, habiendo precisado ingreso hospitalario por cuadros de agudización en múltiples ocasiones. En las exploraciones complementarias: sistemático de sangre: leucocitos 13.300 con fórmula leucocitaria desviada a la izquierda, hematíes 3.780.000, Hb 11,2, Hto 33\%. Bioquímica: glucosa 101, GOT 34, GPT 2, GGT 58, fosfatasa alcalina 308, proteínas totales 6,12 . Rx de tórax (Fig. 1): Infiltrado de características alveolares localizado en lóbulo medio. TAC torácico (Fig. 2): consolidación con broncograma aéreo en ambos lóbulos inferiores y áreas parcheadas en lóbulo medio y ambos lóbulos superiores. Se aprecia así mismo un derrame

Trabajo aceptado: 11 de mayo de 2003

Correspondencia: Félix del Campo Matías. Padre Francisco Suárez, 8, 5 dcha. 47006 Valladolid. e-mail: fsas@eresmas.net 


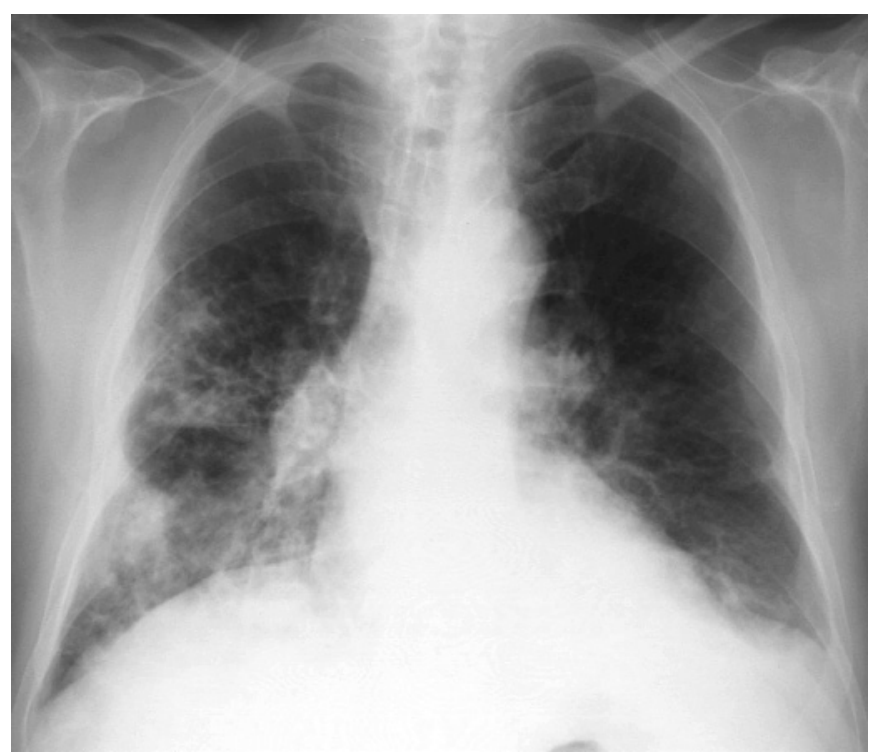

Fig. 1.

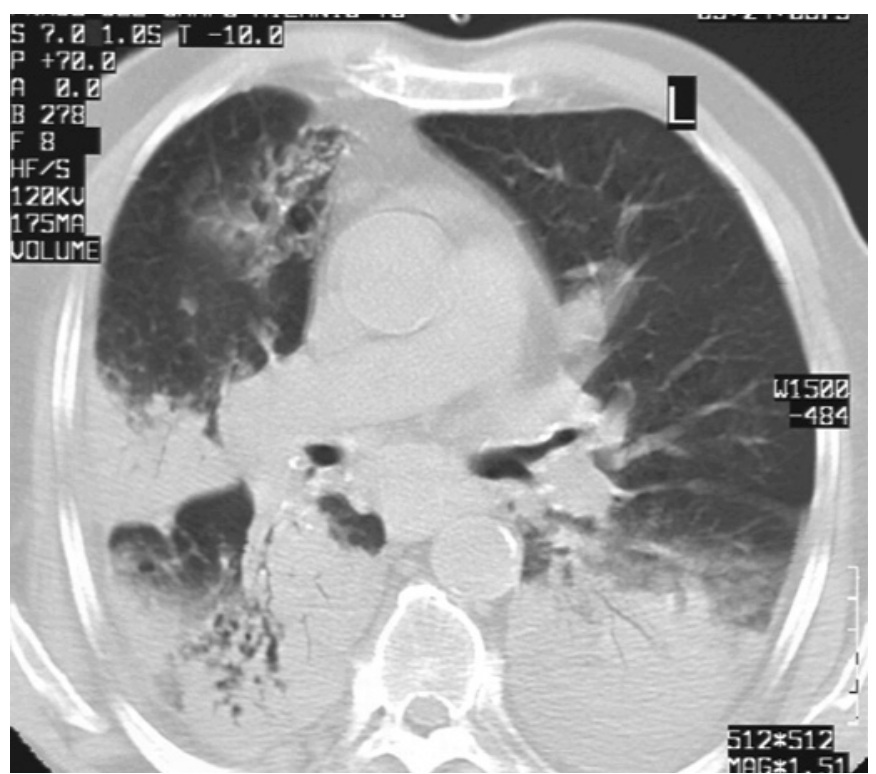

Fig. 2.

pleural bilateral. Gasometría arterial: $\mathrm{PO}_{2} 64, \mathrm{PCO}_{2} 44$, ph 7,45. Hemocultivos negativos. Gram esputo: diplococos gram positivos y bacilos Gram negativos tipo nocardia. Cultivo de esputo: crecimiento de flora normal de vías respiratorias y colonias compatibles con nocardia, siendo identificada posteriormente como nocardia farcínica, realizándose estudio de resistencias. Tinción de Ziehl y cultivo de Lowestein negativo. Serología neumonías atípicas: negativo. Antígeno en orina frente a legionella y streptococus pneumoniae negativo. Ecocardiograma: Normal. Toracocentesis: líquido pleural de aspecto serofibrinoso, que analíticamente corresponde a un exudado, que no evidencio la presencia de gérmenes. Espirometría realizada al alta: FVC 1700 (47\%), FEV1 880 (32\%), FEV1/FVC 52\%.

El paciente fue tratado inicialmente con moxifloxacino con mala respuesta tanto clínica como radiológica, sustituyéndose por ceftriaxona y trimetropin sulfametoxazol al recibir el resultado inicial del cultivo. La respuesta clínica fue desfavorable persistiendo la fiebre. Ante esta situación se inició tratamiento antibiótico con imipenem y amikacina, siendo la evolución clínica y radiológica favorable. El antibiograma realizado confirmó posteriormente la resistencia de la nocardia a la ceftriaxona.

\section{DISCUSIÓN}

La nocardiosis es una enfermedad infrecuente, que suele presentar un curso variable, no existiendo características clínicas, analíticas ni radiológicas patognomónicas. Se trata de bacterias gram positivas, aerobias estrictas, parcialmente acidoalcohol-resistentes y con morfología filamentosa, que pertenecen al orden de los Actinomycetos. La nocardia asteroides, en la actualidad es considerada desde un punto de vista taxonómico, como un grupo bioquímicamente heterogéneo y complejo, de ahí que a estos microorganismos se les denomine como complejo nocardia asteroides, y que incluye tres especies: nocardia asteroides en sentido estricto, nocardia nova y nocardia farcínica ("bacille du farcin") (1).

Se trata de bacterias de carácter ubicuo, que se encuentran ampliamente distribuidas en la naturaleza: suelo, aguas, material vegetal y en una gran variedad de animales. En el hombre, la vía de entrada habitual es la respiratoria como consecuencia de la inhalación, pero también puede ser debida a inoculación directa de la piel. La afectación pulmonar en pacientes con inmunodepresión subyacente es la forma de presentación más común de la nocardiosis en EE.UU. (2), pudiendo originar así mismo infecciones diseminadas, infecciones del sistema nervioso central y afectaciones extrapulmonares localizadas. Las infecciones pulmonares se producen habitualmente por nocardia asteroides. Su incidencia no es bien conocida, habiéndose incrementado en las últimas décadas. En EE.UU. los cálculos estiman que aparecen de 500 a 1.000 casos nuevos por año, de los cuales de un 10 a $15 \%$ de esos pacientes tienen una infección por HIV. La nocardiosis puede tener un carácter de neumonía adquirida en la comunidad pero también se ha descrito como enfermedad nosocomial.

En general, la nocardiosis aparece en enfermos que presentan algún tipo de inmunodepresión (personas con neoplasias, enfermedades sistémicas, transplante médula ósea, transplante de órganos sólidos, tratamiento inmunosupresor, pacientes en tratamiento con corticoides a dosis elevadas, infección por HIV, alcoholismo), pero también ha sido descrito en pacientes inmunocompetentes (3). La afectación pulmonar en pacientes inmunocompetentes es variable, entre un 10$25 \%$ en algunas series alcanzando hasta un $40 \%$. La nocardia puede colonizar el tracto respiratorio de pacientes con patología pulmonar previa: asma, EPOC, etc.

La supresión de la inmunidad celular parece jugar un papel importante en el establecimiento de la infección por nocardia. La inmunidad celular mediante linfocitos $\mathrm{T}$ es efectiva frente a las especies del género Nocardia a nivel pulmonar y previenen la diseminación extrapulmonar de la infección. La disminución de las defensas a nivel local puede predisponer a una afectación pulmonar por nocardia, especialmente en pacientes con enfermedades pulmonares crónicas: EPOC, silicosis, tuberculosis, proteinosis alveolar, etc. $(4,5)$.

En los estudios de revisión este tipo de infección aparece en la mayoría de los casos en pacientes con factores predisponentes entre los que se incluye fundamentalmente tratamientos inmunosupresores, neoplasias, HIV, etc., y más infrecuentemente en pacientes en los que no se recoge ningún factor predisponente. Dentro de la patología pulmonar crónica, la enfermedad pulmonar obstructiva crónica es la que más frecuentemente se describe. Así, Georghiou y Blalock (6) refieren que más del $30 \%$ de sus pacientes con nocardiosis pulmonar tenían entre sus antecedentes el estar diagnosticados de 
EPOC. Menéndez (7) encuentra que tres de los 10 pacientes de su serie presentaban una EPOC, de los cuales uno de ellos había sido tratado con corticoides. Mari (8) en un estudio sobre nocardiosis pulmonar, seis de sus 10 pacientes estaban diagnosticados de EPOC, de los cuales 4 habían recibido corticoterapia crónica, lo que ha inducido a plantear si el factor de riesgo verdadero es la corticoterapia crónica y no la EPOC. Por otra parte, son varios los estudios que consideran que la EPOC se asocia con un mayor riesgo de neumonías adquiridas en la comunidad (9). A su vez, Mari (8) encontró una mayor mortalidad por nocardia pulmonar en los pacientes con EPOC.

La afectación pulmonar puede tener un curso variable, mostrando una evolución fatal en el $10 \%$ de los casos. Puede aparecer a cualquier edad y con una mayor frecuencia en varones. La presentación clínica de la infección por nocardia depende del estado inmune del paciente. En pacientes inmunocompetentes la sintomatología clínica es la habitual de un proceso neumónico: fiebre, tos y expectoración purulenta, sensación de disnea y dolor pleurítico. La nocardiosis pulmonar no tratada presenta una evolución crónica muy similar a la tuberculosis. La afectación pulmonar puede extenderse a la cavidad pleural (10) o diseminarse por vía hematógena, como ocurre en un importante porcentaje de casos. En la analítica de sangre destaca la leucocitosis con neutrofilia. La presentación radiológica es diversa: nódulos únicos o múltiples, masas solitarias, opacidades alveolares que afectan a uno o varios lóbulos e infiltrados reticulonodulares. Las lesiones radiológicas pueden asociarse a cavitación y presencia de derrame pleural (11).

El diagnostico se basa en el aislamiento de la nocardia en las secreciones respiratorias, bien en los cultivos de esputo, en el lavado broncoalveolar o en líquido pleural, y tan solo en pocas ocasiones es necesario recurrir a técnicas invasivas. La falta de sospecha inicial hace que en ocasiones su diagnóstico se retrase. El aislamiento e identificación de la nocardia es complejo. La nocardia crece en medios habituales, pudiendo aparecer dentro de los primeros cuatro días, sin embargo en ocasiones la incubación puede retardarse hasta 2 a 4 semanas.

Solo recientemente se ha reconocido a la nocardia farcínica como un patógeno importante para el ser humano (12), habiendo sido descrita infrecuentemente en la literatura, por las dificultades de su identificación. En los últimos años se han comunicado aisladamente algunos casos en España $(8,13-$ 15), sin embargo su prevalencia es mayor en países como Alemania, en donde alcanza el $43 \%$ de los casos de nocardia asilados. La nocardia farcínica puede dar lugar a infecciones graves: pulmonares, afectación diseminada y del sistema nervioso central, habiendo sido implicada en infecciones adquiridas en la comunidad o con carácter nosocomial. Predomina en varones, asociándose en la mayoría de los casos a patología subyacente $(85 \%)$, siendo la afectación pulmonar la forma de presentación clínica más frecuente.

En el tratamiento de la infección pulmonar por nocardia farcínica se incluye dentro del arsenal terapéutico como antibióticos adecuados la amikacina, imipenem, ciprofloxacino y trimetropin sulfametoxazol, siendo en general resistentes a gentamicina, tobramicina y kanamicina. La nocardia farcínica es la única especie del grupo resistente a las cefalosporinas de tercera generación y a menudo presenta una sensibilidad intermedia frente a fluorquinolonas. La amikacina ha sido utilizada con éxito en combinación con imipenen o cotrimoxazol. Debido a su extrema resistencia frente a los antimicrobianos que se utilizan habitualmente en la terapia de las nocardiosis, hace que sea importante su identificación para un manejo clínico adecuado.

\section{Bibliografía}

1. Lerner PI. Nocardia species. In: Mandell GL, Douglas RG, Bennet JE eds. Pinciples and practice of Infectious Diseases. 2nd Edn. New York: John Wiley \& Sons, 1985, p. 192-206.

2. McNeil MM, Brown JM. The medically important actinomycetes:epidemiology and microbiology. Clin Microbiol Rev 1994; 7: 357-417.

3. Casa A, Camino X, Fernández MC, Resano A, Zubillaga G. Nocardiosis en dos pacientes inmunocompetentes. An Med Interna (Madrid) 2000; 17: 422-424.

4. Beaman BL, Beaman L. Nocardia species:host-parasite relationships. Clin Microbiol Rev 1994; 7: 213-264.

5. Lacassagne L, Didier A, Murris-Espin M, Rouquet RM, Clave D, Lemozy J, Giron J, Leophonte P. Respiratory infection due to nocardia in patients presenting with chronic airflow obstruction. Rev Mal Resp 1996; 13: 433-436

6. Georghiou PR, Blalock ZM. Infection with nocardia species in Queensland. A review of 102 clinical isolates. Med J Aust 1992, 156:

7. Menéndez R, Cordero PJ, Santos M, Gobernado M, Marco V. Pulmonary infection with nocardia species: a report of 10 cases and review. Eur Respir J 1997; 10: 1542-1546.

8. Mari B, Monton C, Mariscal D, Luján M, Sala M, Domingo C. Pulmo- nary nocardiosis:clinical experience in ten cases. Respiration 2001; 68 382-388.

9. Almirall J, Gonzalez C, Balanzo X, Bolivar I. Proportion of community-acquired pneumonia cases attributable to tobacco smoking. Chest 1999; 116: 692-697.

10. Fullana J, García PA, Pellicer P. Absceso pulmonar e hidroneumotórax secundario a infección por nocardia. Arch de Bronconeumol 1999; 35 : 360-361.

11. Feigin DS.Nocardiosis of the lung:chest radiographic finding in 21 cases. Radiology 1986; 159: 9-14.

12. TsukamuraM, Ohta M. Nocardia farcinica as a pathogen of lung infection. Microbiol Inmunol 1989; 24: 237-241.

13. Terraza S, Ramos C, Revillo MJ, Gracia M, Vitoria I, Moles B. Infección pulmonar por nocardia farcínica. Enferm Infecc Microbiol Clin 1999; 17: 258-259.

14. Torres $\mathrm{OH}$. Infection caused by nocardia farcinica:case report and review. Eur J Clin Microbiol Infect Dis 2000; 19: 205-212.

15. De la Iglesia P, Viejo G, Gómez B, de Miguel D, del Valle A, Otero L. Fatal pulmonary nocardia farcinica infection. J Clin Microbiol 2002; 40: 1098-1099. 